OPEN ACCESS

Edited by:

Greg Stuart,

Australian National University,

Australia

Reviewed by:

David Reser,

Monash University, Australia

Marco Mainardi,

Scuola Normale Superiore di Pisa,

Italy

*Correspondence: Trichur R. Vidyasagar trv@unimelb.edu.au

Received: 23 October 2018 Accepted: 14 January 2019 Published: 05 February 2019

Citation:

Vidyasagar TR and Levichkina E (2019) An Integrated Neuronal Model of Claustral Function in Timing the

Synchrony Between Cortical Areas.

Front. Neural Circuits 13:3. doi: 10.3389/fncir.2019.00003

\section{An Integrated Neuronal Model of Claustral Function in Timing the Synchrony Between Cortical Areas}

\author{
Trichur R. Vidyasagar ${ }^{1,2,3 *}$ and Ekaterina Levichkina ${ }^{1,4}$ \\ ${ }^{1}$ Department of Optometry and Vision Science, University of Melbourne, Parkville, VIC, Australia, ${ }^{2}$ Florey Institute of \\ Neuroscience and Mental Health, Parkville, VIC, Australia, ${ }^{3}$ Australian Research Council Centre of Excellence in Integrative \\ Brain Function, University of Melbourne Node, Melbourne, VIC, Australia, ${ }^{4}$ Institute for Information Transmission Problems, \\ Russian Academy of Sciences, Moscow, Russia
}

It has been suggested that the function of the claustrum $(C L)$ may be to orchestrate and integrate the activity of the different cortical areas that are involved in a particular function by boosting the synchronized oscillations that occur between these areas. We propose here a model of how this may be done, thanks to the unique synaptic morphology of the $C L$ and its excitatory and inhibitory connections with most cortical areas. Using serial visual search as an example, we describe how the functional anatomy of the claustral connections can potentially execute the sequential activation of the representations of objects that are being processed serially. We also propose that cross-frequency coupling (CFC) between low frequency signals from $\mathrm{CL}$ and higher frequency oscillations in the cortical areas will be an efficient means of CL modulating neural activity across multiple brain regions in synchrony. This model is applicable to the wide range of functions one performs, from simple object recognition to reading and writing, listening to or performing music, etc.

Keywords: claustrum, neural synchrony, cross-frequency coupling, visual cortex, visual search, attention

\section{A REGION THAT INTEGRATES BRAIN ACTIVITY}

For purposeful and useful interaction with the external world, the brain needs to integrate information processed in different parts of the nervous system, so that it can efficiently process sensory inputs, often from more than one modality, stored memories, emotional aspects of the situation, and executive and motor programmes needed for the chosen response. This requires the operation of many brain areas communicating with each other. Crick and Koch (2005) published a stimulating idea that in the claustrum (CL), the brain may have a central integrator essential for our unified sense of cognition and cohesive behavior. This insight was inspired by the anatomical connectivity between the CL and other brain regions and the synaptic organization within the nucleus itself. The CL connects reciprocally with almost every cortical area (Pearson et al., 1982; Tanné-Gariépy et al., 2002; Druga, 2014; Torgerson et al., 2015; Wang et al., 2017). Furthermore, CL has been found to be the most densely interconnected structure in the human brain (Torgerson et al., 2015), and its internal structure can facilitate rapid development of synchronized activity within adjacent activated regions of the CL (Crick and Koch, 2005; Smythies et al., 2014a; Kim et al., 2016). Crick and Koch (2005) suggested that the dendro-dendritic synapses in the CL, which could potentially include gap junctions (Shepherd and Greer, 1998), can rapidly transfer signals 
arriving from different cortical areas. However, in the only study done in awake behaving macaques specifically aiming to record from multimodal neurones that would support an integrating function for single claustral cells, Remedios et al. (2010) found mainly unimodal sensory cells responding either to visual or auditory stimuli but not to both. Recent rodent studies of claustral circuitry have also shown only very weak connections between principal claustrocortical neurons (Kim et al., 2016). Smythies et al. (2012, 2014a,b) considering a few different hypotheses about how the CL may nevertheless be involved in integrating the activity across many parts of the brain, suggested that the most likely way the CL could exert its integrative function may not be by convergence of signals from various cortical areas on to single claustral cells, but rather by aiding cortical areas to amplify the synchrony between themselves. Saalmann et al. (2012) showed that the pulvinar does a comparable function in the maintenance of a working memory trace in a spatially cued object identification task. They demonstrated that the memory of the object location was maintained by a local cluster of pulvinar cells, as observed in the high degree of local spike-field coherence in the $8-15 \mathrm{~Hz}$ range and leading to almost zero-lag synchrony between visual areas V4 and TEO. This finding was supported by Zhou et al. (2016), who found a similar result prior to the appearance of the stimulus array in their paradigm. These synchronized oscillations were related to the maintenance of a memory trace that would be needed in the immediate future. Could the CL be doing something similar with regard to the actual processing and integration of sensory information and the behavioral response?

\section{CLAUSTRUM COULD ENHANCE SYNCHRONIZED NEURAL OSCILLATIONS BETWEEN CORTICAL AREAS}

A common principle of the mammalian brain that is being recognized as a fundamental mechanism driving its perception, cognition and behavior is the existence of periodic oscillations of neural activity amongst groups of active cells (e.g., Engel et al., 1991; Buzsáki et al., 2013; Buzsáki and Schomburg, 2015). Such rhythmic coordination in excitability is ubiquitous in the brain, but varying in its power, phase and frequency between brain regions and also between tasks. Almost every cortical activity involving processing of sensory information, memory, executive prerogatives and/or behavioral output inevitably engages multiple cortical areas communicating with each other and providing feedforward, feedback or modulatory signals. A plausible mechanism for such inter-areal communication is "communication through coherence" (Bastos et al., 2015), where rhythmic synchronization in one group of neurons leads to modulations in the input gain at synapses that they make on a second group of neurones. Such communication through coherence has been well documented by a number of studies through simultaneous recordings from two different cortical areas in awake macaques performing visual attention tasks (Buschman and Miller, 2007, 2009; Saalmann et al., 2007; Gregoriou et al., 2009).
Smythies et al. (2014a,b) suggested that when two cortical areas that are mutually connected and in a particular task begin to synchronize their activities, their connections to the CL first lead to rapid development of intraclaustral synchrony. These claustral regions are then believed to cause an increase in the synchrony between the two cortical regions through their efferents back to the respective cortical targets. While this proposition addresses the lack of multimodal neurones in the CL and yet ascribes to the CL a central integrative function, it opens up a number of new questions. Most importantly: (1) What is the relationship between the claustrocortical and cortico-cortical synchronies, in particular, do they occur at the same frequency? (2) What is the trigger for getting the CL involved and what terminates the synchrony generated in the cortex by the CL, without letting it evolve into a reverberating or even epileptiform discharge?

\section{OUR HYPOTHESIS OF "PUNCTUATED NEURAL SYNCHRONY"}

In this section, we outline a hypothesis for claustral function and illustrate it by applying it to "serial visual search". Visual search is not only a very common function our brains perform, but is also a widely studied task in both humans and non-human primates. In most variations of this paradigm, one searches for a target among a number of items in a visual scene, with which the target shares one or more features. Early visual search experiments by Treisman and Gelade (1980) led them to propose a "feature integration theory" to explain how we detect objects in a cluttered visual scene and also how we are able to bind the attributes of each object before identification. This highly influential model proposes that a "spotlight of attention" selects at a time one particular object in the visual scene to be processed in detail and then moves on to others until the target is found. As a neural correlate of the feature integration model, it has been proposed (Vidyasagar, 1999; Bullier, 2001) that the dorsal cortical stream and its top-down feedback to the primary visual cortex (area V1) and to ventral stream structures serially select, from a priority map in the posterior parietal cortex, one particular location for a short time (Figure 1A). This is then processed in detail by the ventral areas that deal with object recognition. Despite the functional localization in the primate brain with different areas and neurones being specialized for different attributes such as color and shape, the simultaneous processing of the attributes of only one object at any one time leads to the binding of features of that object alone. In doing this, serial search proceeds at a rate of 20-45 ms/item, depending upon task demands (Wolfe et al., 1998; Wolfe and Horowitz, 2004). This translates into largely a beta and low gamma frequency range $(22.2-50 \mathrm{~Hz})$. The main neurophysiological support for this claim arises from a number of studies: (1) Buschman and Miller (2009, 2010) show that covert shifts of attention in a visual search task is correlated with the cyclical oscillation of top-down prefrontal modulation of parietal activity occurring in the low gamma range; (2) there is a wealth of evidence for the role of lateral intraparietal area (LIP) in directing top-down attention to specific objects 




(Bisley and Goldberg, 2003, 2010; Saalmann et al., 2007; Corbetta and Shulman, 2011; Meehan et al., 2017); (3) experiments in behaving macaques have shown that the top-down attentional feedback modulation of an early visual area, middle temporal (MT or area V5) by the parietal area, LIP is mediated by synchronized oscillations from LIP driving MT neurones at topographically corresponding locations, in the frequency range 25-45 Hz (Saalmann et al., 2007); and (4) though such cyclical modulation has not been directly demonstrated in the dorsal stream feedback to area V1, attentional and contextual modulation of $\mathrm{V} 1$ responses to visual inputs has long been amply demonstrated (Vidyasagar, 1998; Brefczynski and DeYoe, 1999; Ito and Gilbert, 1999; Gandhi et al., 1999; McAdams and Reid, 2005; Vidyasagar and Pigarev, 2007). Given the extensive neurophysiological evidence for synchronized neural oscillations in mediating interareal communication (Buschman and Miller, 2007; Saalmann et al., 2007, 2012; Gregoriou et al., 2009), it is not too speculative to suggest that the feedback to primary visual cortex is also likely to be mediated by such oscillations (Graboi and Lisman, 2003; Vidyasagar, 2013).
Extending the above argument, we propose that the CL's comprehensive reciprocal connections with almost all cortical areas and their unique internal morphology help to magnify the synchrony between cortical areas and also provide a behaviorally useful sequence of activation across the surface of the corresponding cortical areas, such as what is needed in tasks such as serial visual search. Claustral anatomy and its connectivity are likely to accomplish the above requirements. In Figure 1C, we show a simplified canonical circuitry which is applicable to any two or more cortical areas that are functionally connected to the CL in any particular situation, but here shown for a visual task. Taking serial visual search as example, we show on the right claustral efferents projecting to principal (p) cells in both V1 and the dorsal stream (here, marked as LIP/MT).

Afferents to input layers in cortical areas not only synapse on to the excitatory stellate and pyramidal cells, but also to local inhibitory interneurons. Studied most intensively in the primary visual cortex (Creutzfeldt and Ito, 1968; Ferster and Lindström, 1983; LeVay, 1986), such an input leads to a powerful and long-lasting inhibition. Such strong feed forward inhibition 
(FFI) following on the heels of an excitatory input leads to aborting the excitatory response of the target cells after the initial volley (Bruno, 2011). While FFI has been shown to generate oscillations in a local network (Kremkow et al., 2010), FFI from one area to another, here from CL to V1, would serve another additional purpose, namely terminating the initial excitation.

Figure 1B shows how this may function in the case of serial visual search. In a typical visual search task, both engagement and disengagement from the items are essential and furthermore, they should occur sequentially, shifting from one item to another until the target is found, and all of this governed by task priorities. It is now believed (reviewed in Bisley and Goldberg, 2010) that area LIP has a continuously updated priority map that governs the allocation of top-down attentional signal. This priority map itself is updated from a number of inputs-especially task demands as dictated by prefrontal executive areas and saliency of the targets themselves (Ipata et al., 2009; Bisley and Goldberg, 2010). We propose that while the serial engagement of attention is determined simply by the pecking order in the priority map, the disengagement comes from the termination of the synchronized oscillations by the claustrocortical connections with areas that respond to the attributes of the object at the prioritized location. We suggest that such termination and thus the disengagement from the attended item is brought about by the inhibitory volley of the FFI circuit. Since such inhibition is long-lasting, it may also be the neural basis of "inhibition of return" (Wang and Klein, 2010), well-known in the visual search literature. Our proposed role of CL in facilitating top-down attentional modulation is consistent with results of recent experiments in rodents (Mathur, 2014; Goll et al., 2015; Atlan et al., 2018; White et al., 2018). Interestingly, CL not only receives selective top-down attentional influences from the cortex, for example from the anterior cingulate cortex (White et al., 2018), but it also plays a critical role in suppressing auditory distractors in a visual task (Atlan et al., 2018). Such a function is probably related to CL's role in helping to distinguish between relevant and irrelevant items as in a typical search task.

For attentive serial search to work in the fashion described above, we expect that any reciprocal connection from V1 to CL is weak or non-existent. As described earlier, serial search requires moving the spotlight of attention from one item of the scene to another until the target is found. Object recognition is known to occur largely in the ventral stream and it is believed to be facilitated by top-down modulation of incoming visual signals by feedback from the dorsal stream (Vidyasagar, 1999; Bullier, 2001). Once visual attention gets focussed on one object by the spotlight of attention, the CL may play little role in the more detailed processing by the ventral cortical areas. Finding the target would abort the FFI from the CL and the activity in V1 and the corresponding topographic locations in the various cortical areas would continue under focussed attention. Furthermore, if activity related to object locations are supposed to be "serially highlighted" for further processing by extra-striate areas such as LIP, MT, V4 and TEO for ultimate binding of the attributes of the object, such a scheme would be defeated if there are strong signals from every item to the CL, triggering reciprocal synchronizing volleys. In fact, many studies on the $\mathrm{CL}$, while describing the widespread afferent connections from the CL to most association areas and the prefrontal cortex have emphasized the uncertainty of the projection from the primary sensory areas, including V1 in the primate (Druga, 2014; reviewed in Smythies et al., 2014a). There is also a cautionary note about the effectiveness of the V1 (area 17) to CL projection that has been described in the cat. LeVay and Sherk (1981), who studied connections between visual areas and CL in the cat, found that area 17 cells projecting to CL were just $3.5 \%$ of layer 6 cells and these were found predominantly in the peripheral rather than central visual field representation, whereas the claustral projections to area 17 were much heavier. Sherman and Guillery (2011) state that the layer 6 cells that project subcortically are class 2 glutamatergic cells that do not produce much spiking activity but only modulate responses mainly through metabotropic postsynaptic receptors. Thus, the claustral synchrony may get initiated and sustained, not so much by the sensory input to primary sensory areas, but rather by activity in higher areas such as LIP. Thereafter, as the enhanced synchrony between the representations of a particular object in different cortical areas (in Figure 2, V1 and LIP/MT) develops and then dies down with its termination by FFI, the next most salient location in LIP synchronizes with V1 and the corresponding locations in the CL also get activated and a new cycle of enhanced synchrony starts, to be in its turn terminated by the subsequent FFI.

Recent studies of the rodent CL have demonstrated the strong inhibitory influence that optogenetic stimulation of claustral outputs could have on cortical areas, namely on unit responses in the anterior cingulate cortex (White et al., 2018), the prefrontal cortex (Jackson et al., 2018; Narikiyo et al., 2018) and the auditory cortex (Atlan et al., 2018). We suggest that these inhibitory volleys represent the FFI needed to terminate activity in target areas as described above in our scheme. It is noteworthy that in all of these studies, the optogenetic excitation was

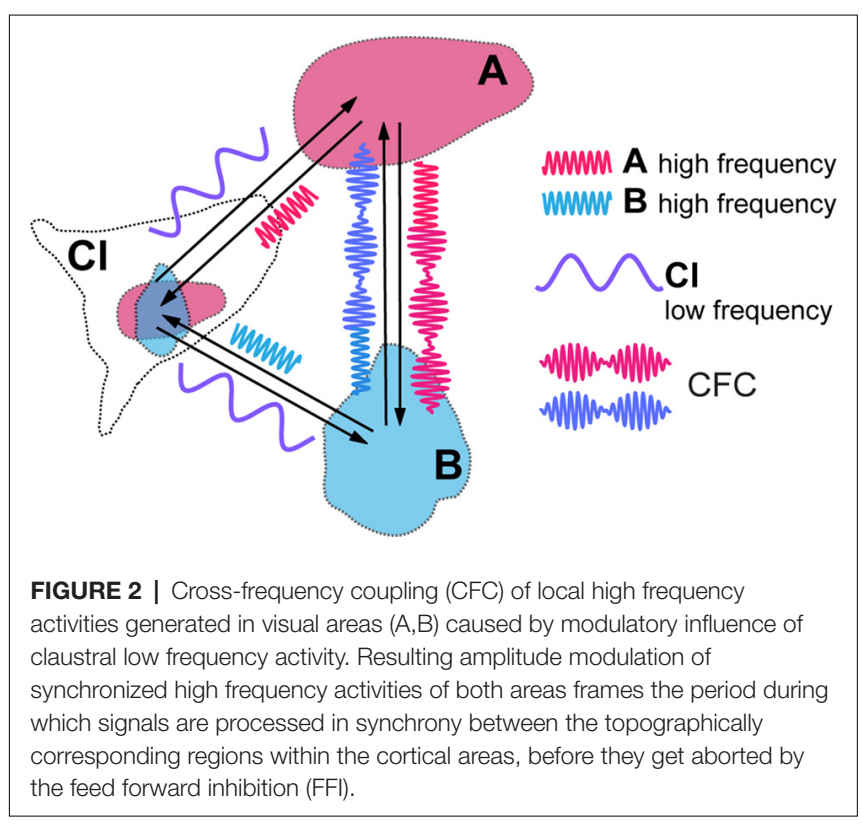


effective in causing the inhibition in target cortical areas, but the initial excitatory response in the target area was rather weak (Narikiyo et al., 2018). This may be attributed to two factors: (i) the optogenetic stimulation does not resemble the usual synchronized oscillatory activity that may be needed to cause the excitatory oscillations as described above and in the section below on cross-frequency coupling (CFC) and (ii) the excitatory response would require temporal simultaneity of oscillatory inputs from other cortical areas.

Recent rodent studies have also elucidated a claustral circuitry that could be ideally suited to our proposed function of the CL (Kim et al., 2016, see their Figure 8), by possibly enabling another FFI circuit within the CL itself. While corticoclaustral inputs target individual claustrocortical (ClaC) cells monosynaptically and there are few direct connections among these principal, claustrocortical cells, the cortical inputs to $\mathrm{CL}$ provide strong stimulation to the parvalbumin (PV)positive inhibitory interneurons, which are themselves strongly interconnected via both electrical and chemical synapses (Kim et al., 2016). This leads to a situation where synchronous activation signals from two different cortical areas to their reciprocally connected $\mathrm{ClaC}$ cells would set off a neural synchrony between the cortical areas and the CL, soon to be followed by an inhibitory volley that suppresses the claustral outputs.

Finally, when the target in a visual search task is found, the termination of all activity in the CL and the search itself may be brought about by stimulation of the kappa opioid receptors, the mRNA for which is particularly plentiful in the CL (Mansour et al., 1994). The high density of these receptors on claustral cells is a striking finding that needs particular consideration in any model of claustral function. The possible role of this receptor system in the larger integrative functions has been pointed out (Stiefel et al., 2014), since such receptor stimulation inhibits the release of GABA (Hjelmstad and Fields, 2003; Li et al., 2012) which in turn would disrupt the generation of oscillations within the CL and the claustral amplification of the synchrony between cortical areas. Activation of the kappa receptors inhibits both glutamate and GABA transmission (Hjelmstad and Fields, 2003), thus practically stopping excitatory activity as well as disrupting oscillations. We believe that a match between an object brought under the roving spotlight of attention and the representation of the expected object may abort the visual search through its effect on claustral kappa opioid receptors. While the kappa opioid system may be generally known for its dysphoric effects, particularly in producing the aversive and depressive effects in the case of drug abuse (Lalanne et al., 2014), the evolutionary reason for the kappa receptors are not likely to be related to drug addiction. Natural opioids acting on mu opioid receptor (MOR) and kappa receptors are known to lead to opposing effects in rats performing a behavioral task, the former to reinforcement of the related behavior and the latter to its termination (Shippenberg and Herz, 1986). Though stimulation of kappa receptors in the ventral tegmental area may be related to motivational and hedonic aspects (Spanagel et al., 1992), similar stimulation in other areas may have effects depending upon the function of those respective areas.
Thus, their primary role may be simply in aborting neural synchrony in local circuits through their action on GABEergic transmission, besides the inhibition of the excitatory activity itself. We propose that until the visual search is completed, there is little stimulation of the claustral kappa-opioid receptors, but a specific input to the CL on finding the target, possibly from the prefrontal regions which are heavily linked to the CL (Reser et al., 2014), may disrupt neural oscillations in the CL and consequently its amplification of synchrony in various cortical regions.

Our model of claustral control of visual search is one convenient example for what we believe to be a description of claustral function in general. We believe that the proposed role of $\mathrm{CL}$ in sequencing neuronal activity is not restricted to the visual modality, but in line with its widespread cortical connections, CL can potentially modulate activity in all sensory cortices, association areas and also motor areas. Thus, we hypothesize that the CL might be instrumental in not only in binding the activity of different cortical regions by enhancing their synchrony, but also organizing all cortex-mediated processes in a sequential manner, as for example in language comprehension, language production and in organizing complex motor programs.

\section{CLAUSTRAL MODULATION OF OTHER BRAIN AREAS THROUGH CROSS-FREQUENCY COUPLING}

CFC is being recognized as an efficient means of communication between two cortical areas and it is likely to play a critical role in mediating working memory and in enabling learning (Canolty and Knight, 2010; Lisman and Jensen, 2013; Hyafil et al., 2015). Blood-oxygen-level dependent (BOLD) connectivity between areas is best predicted by low frequency oscillations that determine the amplitude of gamma frequencies (Wang et al., 2012). Thus, in the above example, in target cortical areas such as LIP, MT and V1, the amplitudes of a higher, such as high beta or gamma, frequency rhythm may be modulated by, and thus nested within, a lower frequency, for instance theta, alpha or low beta, at which claustral efferents send out their modulating signals to their targets (Figure 2). We expect that each cycle of the low frequency signal from CL would allow sufficient number of high frequency cycles at its target areas to synchronize before the excitatory volley gets aborted by the FFI. While electrical stimulation of lateral geniculate nucleus (LGN) leads to disynaptically mediated inhibitory post-synaptic potentials in stellate cells in layer 4 of the primary visual cortex within a few milliseconds (Creutzfeldt and Ito, 1968; Ferster and Lindström, 1983), with visual stimulation the inhibition seen in intracellular recordings from the cat striate cortex develops gradually over many tens of milliseconds (Pei et al., 1994; Volgushev et al., 1995: Ringach et al., 1997). Both with such visual stimulation and with electrical stimulation (Viswanathan et al., 2011), the inhibition can however last many hundreds of milliseconds. Strong FFI caused by CL stimulation and mediated in vivo by relatively slow neuropeptide $\mathrm{Y}$ interneurons was also described in the prefrontal areas of rodents by Jackson et al. (2018), with the 
excitation/inhibition ratio of cortical pyramidal cells equalling just 0.25 . Though one is yet to see similar studies done in the case of the primate $\mathrm{CL}$, the window of opportunity for neural synchrony between relevant cortical regions to be amplified by claustral output is likely to be defined by the time course of the FFI circuit. It is possible that this time course may also be modulated by task demands and the state of vigilance.

The cyclical facilitation of processing of incoming visual signals in V1 would mean that sensitivity to visual stimuli could show periodic fluctuation, as indeed they do (Busch et al., 2009; Mathewson et al., 2009; VanRullen and Dubois, 2011). CFC with nested frequencies may also be critical for processing of stimuli at multiple temporal rates, such as graphemes/phonemes, and syllables and words during reading and speech perception (Graboi and Lisman, 2003; Vidyasagar, 2013). Through CFC, claustral output at one low frequency (delta, theta, alpha, or low beta) can modulate a range of oscillation frequencies (high beta or gamma) at cortical areas that are connected to each other in a task such as reading or visual search. Figure $\mathbf{2}$ is a simplified diagram of how this might function in the case of CL boosting synchrony between LIP/MT and V1. At this stage, it is too premature to speculate at what frequency the claustral assembly oscillates. It may be either always at the same frequency which is determined by its own morphology and resonance frequency or dictated by the area that triggers the synchrony in the first place or even under an executive command from the prefrontal cortex.

\section{OUTSTANDING QUESTIONS FOR FUTURE STUDIES}

The model leads to a number of testable predictions. The following are some of the main questions for study.

1. In tasks such as visual search, the model predicts neural activity in CL driving synchronized activities in relevant cortical regions.

2. Claustral influence on cortical regions would exhibit two stages: an initial excitatory oscillation followed by strong inhibition.

3. The time course of the FFI from CL on cortical areas needs to be ascertained to test whether it permits synchrony between cortical areas.

4. Is the low frequency volley from CL mediating CFC fixed or is it dynamically modified by task demands?

5. In a visual search task, is there a roving wave of synchrony across the CL as the animal performs a search task, as the corresponding topographic locations in the CL serially facilitate the scan of the spotlight of attention?

\section{REFERENCES}

Atlan, G., Terem, A., Peretz-Rivlin, N., Sehrawat, K., Gonzales, B. J., Pozner, G., et al. (2018). The claustrum supports resilience to distraction. Curr. Biol. 28, 2752.e7-2762.e7. doi: 10.1016/j.cub.2018.06.068

Bastos, A. M., Vezoli, J., and Fries, P. (2015). Communication through coherence with inter-areal delays. Curr. Opin. Neuribiol. 31, 173-180. doi: 10.1016/j.conb. 2014.11.001
6. Is there a rapid termination of intraclaustral synchrony and stimulation of GABAergic neurons as soon as the target is found?

Some of these questions need to be addressed in awake non-human primates. So far, with rare exceptions (Remedios et al., 2010, 2014) the primate CL has defied functional studies, due to its shape and anatomical location, but it is possible that with newer emerging techniques, the experiments are feasible.

\section{CONCLUSION}

Our hypothesis suggests the existence of a functional circuit by which CL could play a vital role in communication between cortical areas by enhancing both the synchrony between cortical areas as well the amplitude of oscillations. The scheme has the advantage that though the connections between cortical areas themselves may not be structurally and functionally strong to develop enough synchrony, the boost given by the CL can help them to attain a degree of synchrony that will be functionally useful. Critical to this function is the unique claustral morphology (Kim et al., 2016) and the FFI circuit both within the CL and in its cortical targets, which are features considered to be characteristic of a system designed to amplify correlated neuronal activity (Bruno, 2011; Hu et al., 2014). The metaphor that Crick and Koch (2005) thought of, that the CL is like the conductor of an orchestra, is apt in more ways than one. In short, the punctuated synchrony we propose is akin to the conductor of an orchestra co-ordinating and inspiring a harmonious and smoothly punctuated symphony. In short, it is a conductor of the synchrony between cortical areas.

\section{AUTHOR CONTRIBUTIONS}

TV was responsible for the basic idea proposed in the article and drafting the first version. He also conducted some of the experiments that underpin crucial elements of the proposed theory. EL assisted in developing the basic idea to fit into a diagrammatic scheme and provided critical input towards the article's intellectual content. EL also enhanced comprehension of the article with revisions and the illustrations.

\section{FUNDING}

National Health and Medical Research Council (NHMRC) supported some of the critical experiments of TV that form the basis of this article. Australian Research Council (ARC) Centre of Excellence for Integrative Brain Function funded the publication charges.

Bisley, J. W., and Goldberg, M. E. (2003). Neuronal activity in the lateral intraparietal area and spatial attention. Science 299, 81-86. doi: 10.1126/science. 1077395

Bisley, J. W., and Goldberg, M. E. (2010). Attention, intention and priority in the parietal lobe. Annu. Rev. Neurosci. 33, 1-21. doi: 10.1146/annurev-neuro060909-152823

Brefczynski, J. A., and DeYoe, E. A. (1999). A physiological correlate of the “spotlight" of visual attention. Nat. Neurosci. 2, 370-374. doi: 10.1038/7280 
Bruno, R. M. (2011). Synchrony in sensation. Curr. Opin. Neurobiol. 21, 701-708. doi: 10.1016/j.conb.2011.06.003

Bullier, J. (2001). Integrated model of visual processing. Brain Res. Brain Res. Rev. 36, 96-107. doi: 10.1016/s0165-0173(01)00085-6

Busch, N. A., Dubois, J., and VanRullen, R. (2009). The phase of ongoing EEG oscillations predicts visual perception. J. Neurosci. 29, 7869-7876. doi: 10.1523/JNEUROSCI.0113-09.2009

Buschman, T. J., and Miller, E. K. (2007). Top-down versus bottom-up control of attention in the prefrontal and posterior parietal cortices. Science 315, 1860-1862. doi: 10.1126/science.1138071

Buschman, T. J., and Miller, E. K. (2009). Serial, covert shifts of attention in visual search are reflected by the frontal eye fields and correlated with population oscillations. Neuron 63, 386-396. doi: 10.1016/j.neuron.2009.06.020

Buschman, T. J., and Miller, E. K. (2010). Shifting the spotlight of attention: evidence for discrete computations in cognition. Front. Hum. Neurosci. 4:194. doi: 10.3389/fnhum.2010.00194

Buzsáki, G., and Schomburg, E. V. (2015). What does gamma coherence tell us about inter-regional neural communication? Nat. Neurosci. 18, 484-489. doi: $10.1038 / \mathrm{nn} .3952$

Buzsáki, G., Logothetis, N., and Singer, W. (2013). Scaling brain size, keeping timing: evolutionary preservation of brain rhythms. Neuron 80, 751-764. doi: 10.1016/j.neuron.2013.10.002

Canolty, R. T., and Knight, R. T. (2010). The functional role of cross-frequency coupling. Trends Cogn. Sci. 14, 506-515. doi: 10.1016/j.tics.2010.09.001

Corbetta, M., and Shulman, G. L. (2011). Spatial neglect and attention networks. Annu. Rev. Neurosci. 34, 569-599. doi: 10.1146/annurev-neuro-061010-113731

Creutzfeldt, O. D., and Ito, M. (1968). Functional synaptic organization of primary visual cortex neurones in the cat. Exp. Brain Res. 6, 324-352. doi: $10.1007 / \mathrm{bf} 00233183$

Crick, F. C., and Koch, C. (2005). What is the function of the claustrum? Phil. Trans. Roy. Soc. Lond. B Biol. Sci. 36, 1271-1279. doi: 10.1098/rstb.2005.1661

Druga, R. (2014). "The structure and connections of the claustrum," in The Claustrum, ed. J. Smythies (San Diego: Elsevier), 29-84.

Engel, A. K., König, P., Kreiter, A. K., and Singer, W. (1991). Interhemispheric synchronization of oscillatory neuronal responses in cat visual cortex. Science 88, 1177-1179. doi: 10.1126/science.252.5009.1177

Ferster, D., and Lindström, S. (1983). An intracellular analysis of geniculo-cortical connectivity in area 17 of the cat. J. Physiol. 342, 181-215. doi: 10.1113/jphysiol. 1983.sp014846

Gandhi, S. P., Heeger, D. J., and Boynton, G. M. (1999). Spatial attention affects brain activity in human primary visual cortex. Proc. Nat. Acad. Sci. U S A 96, 3314-3319. doi: 10.1073/pnas.96.6.3314

Goll, Y., Atlan, G., and Citri, A. (2015). Attention: the claustrum. Trends Neurosci. 38, 486-495. doi: 10.1016/j.tins.2015.05.006

Graboi, D., and Lisman, J. (2003). Recognition by top-down and bottom-up processing in cortex: the control of selective attention. J. Neurophysiol. 90, 798-810. doi: 10.1152/jn.00777.2002

Gregoriou, G. G., Gotts, S. J., Zhou, H., and Desimone, R. (2009). Long-range neural coupling through synchronization with attention. Science 176, 35-45. doi: 10.1016/s0079-6123(09)17603-3

Hjelmstad, G. O., and Fields, H. L. (2003). Kappa opioid receptor activation in the nucleus accumbens inhibits glutamate and GABA release through different mechanisms. J. Neurophysiol. 89, 2389-2395. doi: 10.1152/jn.01115.2002

Hu, H., Gab, J., and Jonas, P. (2014). Interneurons, fast-spiking, parvalbumin+ GABAergic interneurons: from cellular design to microcircuit function. Science 345:1255263. doi: $10.1126 /$ science. 1255263

Hyafil, A., Giraud, A. L., Fontolan, L., and Gutkin, B. (2015). Neural crossfrequency coupling: connecting architectures, mechanisms and functions. Trends Neurosci. 38, 725-740. doi: 10.1016/j.tins.2015.09.001

Ipata, A. E., Gee, A. L., Bisley, J. W., and Goldberg, M. E. (2009). Neurons in the lateral intraparietal area create a priority map by the combination of disparate signals. Exp. Brain Res. 192, 479-488. doi: 10.1007/s00221-008-1557-8

Ito, M., and Gilbert, C. D. (1999). Attention modulates contextual influences in the primary visual cortex of alert monkeys. Neuron 22, 593-604. doi: 10.1016/s0896-6273(00)80713-8

Jackson, J., Karnani, M. M., Zemelman, B. V., Burdakov, D., and Lee, A. K. (2018). Inhibitory control of prefrontal cortex by the claustrum. Neuron. 99, 1029.e4-1039.e4. doi: 10.1016/j.neuron.2018.07.031
Kim, J., Matney, C. J., Roth, R. H., and Brown, S. P. (2016). Synaptic organization of the neuronal circuits of the claustrum. J. Neurosci. 36, 773-784. doi: 10.1523/jneurosci.3643-15.2016

Kremkow, J., Perrinet, L. U., Masson, G. S., and Aertsen, A. (2010). Functional consequences of correlated excitatory and inhibitory conductances in cortical networks. J. Comput. Neurosci. 28, 579-594. doi: 10.1007/s10827-010-0240-9

Lalanne, L., Ayranci, G., Kieffer, B. L., and Lutz, P. E. (2014). The kappa opioid receptor: from addiction to depression and back. Front. Psychiatry 5:170. doi: $10.3389 /$ fpsyt.2014.00170

LeVay, S. (1986). Synaptic organization of claustral and geniculate afferents to the visual cortex of the cat. J. Neurosci. 6, 3564-3575. doi: 10.1523/jneurosci.06-1203564.1986

LeVay, S., and Sherk, H. (1981). The visual claustrum of the cat. I. Structure and connections. J. Neurosci. 1, 956-980. doi: 10.1126/science.7209525

Li, C., Pleil, K. E., Stamatakis, A. M., Busan, S., Vong, L., Lowell, B. B., et al. (2012). Presynaptic inhibition of gamma-aminobutyric acid release in the bed nucleus of stria terminalis by kappa opioid receptor signalling. Biol. Pysch. 71, 725-732. doi: 10.1016/j.biopsych.2011.11.015

Lisman, J. E., and Jensen, O. (2013). The Theta-Gamma neural code. Neuron 77, 1002-1016. doi: 10.1016/j.neuron.2013.03.007

Mansour, A., Fox, C. A., Burke, S., Meng, F., Thompson, R. C., Akil, H., et al. (1994). Mu, delta and kappa opioid receptor mRNA expression in the rat CNS: an in situ hybridization study. J. Comp. Neurol. 350, 412-438. doi: 10.1002/cne. 903500307

Mathewson, K. E., Gratton, G., Fabiani, M., Beck, D. M., and Ro, T. (2009). To see or not to see: prestimulus alpha phase predicts visual awareness. J. Neurosci. 29, 2725-2732. doi: 10.1523/jneurosci.3963-08.2009

Mathur, B. N. (2014). The claustrum in review. Front. Syst. Neurosci. 8:48. doi: 10.3389 /fnsys.2014.00048

McAdams, C. J., and Reid, R. C. (2005). Attention modulates the responses of simple cells in monkey primary visual cortex. J. Neurosci. 25, 11023-11033. doi: 10.1523/JNEUROSCI.2904-05.2005

Meehan, T. P., Bressler, S. L., Tang, W., Astafiev, S. V., Sylvester, C. M., Shulman, G. L., et al. (2017). Top-down cortical interactions in visuospatial attention. Brain Struct. Funct. 222, 3127-3145. doi: 10.1007/s00429-0171390-6

Narikiyo, K., Mizuguchi, R., Ajima, A., Mitsui, S., Shiozaki, M., Hamanaka, H., et al. (2018). The claustrum coordinates cortical slow-wave activity. BioRxiv [preprint]. doi: 10.1101/286773

Pearson, R. C. A., Brodal, P., Gatter, K. C., and Powell, T. P. (1982). The organization of the connections between the cortex and the claustrum in the monkey. Brain Res. 234, 435-441. doi: 10.1016/0006-8993(82)90883-6

Pei, X., Vidyasagar, T. R., Volgushev, M., and Creutzfeldt, O. D. (1994). Receptive field analysis and orientation selectivity of postsynaptic potentials of simple cells in cat visual cortex. J. Neurosci. 14, 7130-7140. doi: 10.1523/jneurosci.1411-07130.1994

Remedios, R., Logothetis, N. K., and Kayser, C. (2010). Unimodal responses prevail in multisensory claustrum. J. Neurosci. 30, 12902-12907. doi: $10.1523 /$ jneurosci.2937-10.2010

Remedios, R., Logothetis, N. K., and Kayser, C. (2014). A role of the claustrum in auditory scene analysis by reflecting sensory change. Front. Syst. Neurosci. 8:44. doi: 10.3389/fnsys.2014.00044

Reser, D. H., Richardson, K. E., Montibeller, M. O., Zhao, S., Chan, J. M., Soares, J. G., et al. (2014). Claustrum projections to prefrontal cortex in the capuchin monkey (Cebus apella). Front. Syst. Neurosci. 8:123. doi: $10.3389 /$ fnsys.2014.00123

Ringach, D. L., Hawken, M. J., and Dhapley, R. M. (1997). Dynamics of orientation tuning in macaque primary visual cortex. Nature 387, 281-284. doi: $10.1038 / 387281 \mathrm{a} 0$

Saalmann, Y. B., Pigarev, I. N., and Vidyasagar, T. R. (2007). Neural mechanisms of visual attention: how top-down feedback highlights relevant locations. Science 316, 1612-1615. doi: 10.1126/science.1139140

Saalmann, Y. B., Pinsk, M. A., Wang, L., Li, X., and Kastner, S. (2012). The pulvinar regulates information transmission between cortical areas based on attention demands. Science 337, 753-756. doi: 10.1126/science.1223082

Shepherd, G. M., and Greer, C. A. (1998). "Olfactory bulb," in The Synaptic Organization of the Brain, ed. G. M. Shepherd (New York, NY, USA: Oxford University Press), 159-204. 
Sherman, S. M., and Guillery, R. W. (2011). Distinct functions for direct and transthalamic corticocortical connections. J. Neurophysiol. 106, 1068-1077. doi: $10.1152 /$ jn. 00429.2011

Shippenberg, T. S., and Herz, A. (1986). Differential effects of mu and kappa opioid systems on motivational processes. NIDA Res. Monogr. 75, 563-566. doi: 10.1037/e471872004-001

Smythies, J., Edelstein, L., and Ramachandran, V. (2012). Hypotheses relating to the function of the claustrum. Front. Integr. Neurosci. 6:53. doi: 10.3389/fnint. 2012.00053

Smythies, J., Edelstein, L., and Ramachandran, V. (2014a). "Hypothesis relating to the function of the claustrum," in The Claustrum, ed. J. Smythies (San Diego: Elsevier), 299-352.

Smythies, J., Edelstein, L., and Ramachandran, V. (2014b). Hypothesis relating to the function of the claustrum II: does the claustrum use frequency codes? Front Integr Neurosci. 8:7. doi: 10.3389/fnint.2014.00007

Spanagel, R., Herz, A., and Shippenberg, T. S. (1992). Opposing tonically active endogenous opioid systems modulate the mesolimbic dopaminergic pathway. Proc. Natl. Acad. Sci. U S A 89, 2046-2050. doi: 10.1073/pnas.89.6.2046

Stiefel, K. M., Merrifield, A., and Holcombe, A. O. (2014). Claustrum's proposed role in consciousness is supported by the effect and target localization of saliva divinorum. Front. Integr. Neurosc. 26, 8-20. doi: 10.3389/fnint. 2014.00020

Tanné-Gariépy, J., Boussaoud, D., and Rouiller, E. M. (2002). Projections of the claustrum to the primary motor, premotor and prefrontal cortices in the macaque monkey. J. Comp. Neurol. 454, 140-157. doi: 10.1002/cne.10425

Torgerson, C. M., Irimia, A., Goh, S. Y., and Van Horn, J. D. (2015). The DTI connectivity of the human claustrum. Hum. Brain Mapp. 36, 827-838. doi: $10.1002 / \mathrm{hbm} .22667$

Treisman, A. M., and Gelade, G. (1980). A feature-integration theory of attention. Cogn. Psych. 12, 97-136. doi: 10.1016/0010-0285(80)90005-5

VanRullen, R., and Dubois, J. (2011). The psychophysics of brain rhythms. Front. Psychol. 2:203. doi: 10.3389/fpsyg.2011.00203

Vidyasagar, T. R. (1998). Gating of neuronal responses in macaque primary visual cortex by an attentional spotlight. Neuroreport 9, 1947-1952. doi: 10.1097/00001756-199806220-00006

Vidyasagar, T. R. (1999). A neuronal model of attentional spotlight: parietal guiding the temporal. Brain Res. Rev. 30, 66-76. doi: 10.1016/s01650173(99)00005-3

Vidyasagar, T. R. (2013). Reading into neuronal oscillations in the visual system: implications for developmental dyslexia. Front. Hum. Neurosci. 7:811. doi: $10.3389 /$ fnhum.2013.00811
Vidyasagar, T. R., and Pigarev, I. N. (2007). Gating of neuronal responses in macaque primary visual cortex in a memory task. Eur. J. Neurosci. 25, 2547-2557.

Viswanathan, S., Jayakumar, J., and Vidyasagar, T. R. (2011). Role of feedforward geniculate inputs in the generation of orientation selectivity in the cat's primary visual cortex. J. Physiol. 589, 2349-2361. doi: 10.1113/jphysiol.2010.202317

Volgushev, M., Vidyasagar, T. R., and Pei, X. (1995). Dynamics of the orientation tuning of postsynaptic potentials in the cat visual cortex. Vis. Neurosci. 12, 621-628. doi: 10.1017/s0952523800008919

Wang, L., Saalmann, Y. B., Pinsk, M. A., Arcaro, M. J., and Kastner, S. (2012). Electrophysiological low-frequency coherence and cross-frequency coupling contribute to BOLD connectivity. Neuron 76, 1010-1020. doi: 10.1016/j. neuron.2012.09.033

Wang, Q., Ng, L., Harris, J. A., Feng, D., Li, Y., Royall, J. J., et al. (2017). Organization of the connections between claustrum and cortex in the mouse. J. Comp. Neurol. 525, 1317-1346. doi: 10.1002/cne.24047

Wang, Z., and Klein, R. M. (2010). Searching for inhibition of return in visual search. Vis. Res. 50, 220-228. doi: 10.1016/j.visres.2009.11.013

White, M. G., Panicker, M., Mu, C., Carter, A. M., Roberts, B. M., Dharmasri, P. A., et al. (2018). Anterior cingulate cortex input to the claustrum is required for top-down action control. Cell Rep. 22, 84-95. doi: 10.1016/j.celrep.2017.12.023

Wolfe, J. M., and Horowitz, T. S. (2004). What attributes guide the deployment of visual attention and how do they do it? Nat. Rev. Neurosci. 5, 495-501. doi: 10.1038/nrn1411

Wolfe, J. M., O’Neill, P., and Bennett, S. C. (1998). Why are there eccentricity effects in visual search? Visual and attentional hypotheses. Percept. Psychophys. 60, 140-156. doi: 10.3758/bf03211924

Zhou, H., Schafer, R. J., and Desimone, R. (2016). Pulvinar-Cortex Interactions in Vision and Attention. Neuron 89, 209-220. doi: 10.1016/j.neuron.2015.11.034

Conflict of Interest Statement: The authors declare that the research was conducted in the absence of any commercial or financial relationships that could be construed as a potential conflict of interest.

Copyright (c) 2019 Vidyasagar and Levichkina. This is an open-access article distributed under the terms of the Creative Commons Attribution License (CC BY). The use, distribution or reproduction in other forums is permitted, provided the original author(s) and the copyright owner(s) are credited and that the original publication in this journal is cited, in accordance with accepted academic practice. No use, distribution or reproduction is permitted which does not comply with these terms. 